\title{
Defining the vulnerable patient with myeloma-a frailty position paper of the European Myeloma Network
}

\author{
Gordon Cook $\mathbb{D}^{1} \cdot$ Alessandra Larocca ${ }^{2} \cdot{\text { Thierry } \text { Facon }^{3} \cdot \text { Sonja Zweegman }}^{4} \cdot$ Monika Engelhardt $^{5}$
}

Received: 20 April 2020 / Revised: 4 June 2020 / Accepted: 8 June 2020 / Published online: 18 June 2020

(c) Springer Nature Limited 2020

\begin{abstract}
As the treatment landscape continues to evolve towards the application of precision medicine in multiple myeloma (MM), there is a clear need to identify those patients who are at risk of not achieving the maximum benefit whilst exposed to the highest level of toxicity. This group of patients, defined as frail, is an unmet clinical need. However, how we define such a vulnerable group of patients with MM remains to be clarified. An integral aspect of this is to define the physiological age and capacity of patients with MM to deal with the burden of their disease and it's treatment. Such assessments may include not only functional and clinical assessments but also laboratory-based biomarkers of frailty, aging and senescent cellular burden. A need to develop, test and validate clinical screening scores before their adoption into clinical practice is mandated. This position paper from the European Myeloma Network aims to review what is known about defining frailty in MM, and how we can advance this knowledge for the design of clinical trials and ultimately how we deliver treatment in the clinic.
\end{abstract}

\section{Introduction-the unmet need}

The incidence and prevalence of cancer among older adults will increase dramatically over the next 30 years in large part because the elderly population is growing. It has been estimated that the global cancer incidence in the older person will rise from 6.7 million in 2012 to a projected 14 million by 2035 [1]. It is estimated that $70 \%$ of cancers will occur in patients older than 65 years by the year 2030. The decision to treat older adults with cancer should not be based on chronologic age alone. Multiple Myeloma (MM)

Gordon Cook

g.cook@leeds.ac.uk

1 Leeds Institute of Clinical Trial Research \& Leeds Cancer Centre, University of Leeds, Leeds, UK

2 Myeloma Unit, Division of Hematology, University of Torino, Azienda Ospedaliero-Universitaria Città della Salute e della Scienza, Torino, Italy

3 CHU Lille, Service des Maladies du Sang, F-59000 Lille, France

4 Department of Haematology, Amsterdam UMC, Vrije Universiteit Amsterdam, Cancer Center Amsterdam, Amsterdam, The Netherlands

5 Medical Department, Hematology, Oncology \& Stem Cell Transplantation, Clinical Cancer Research Group, Freiburg, Faculty of Freiburg, Freiburg, Germany is the second most common haematological malignancy with almost 5000 patients diagnosed in the UK each year and over 35,000 diagnosed in Europe in 2016 alone [2, 3]. $\mathrm{MM}$ is predominantly a disease of older people, with twothirds of patients aged over 70 at diagnosis. As such the incidence is increasing as the population ages. Furthermore, the increasing age at presentation, associated with agerelated systemic changes as well as co-morbidities has been shown to be related to a higher frequency of treatment discontinuation and non-haematological adverse event $[2,4,5]$.

Over the last 10 years the development of novel biological agents for the management of MM (proteasome inhibitors-PI and immunomodulatory drugs-ImiDs, amongst others) has improved outcomes for patients with MM such that the median overall survival is more than 7-8 years for younger/fitter patients. However, the impact of these therapies has been less marked in the older/less fit transplant non-eligible (TNE) population, particularly those over the age of 75 years [6]. These patients do not have a greater incidence of molecularly high-risk disease and so these differences are likely to be accounted for by differences in patient physiology, increased treatment-related toxicity limiting delivery of effective therapy and less effective, rigorous anti-myeloma treatment being given (undertreatment) [7]. Life expectancy is extremely variable in the same age group, thus suggesting that not only 
chronological age is important but also the health status of the patient [8]. This group therefore has a high un-met need both for new, less toxic treatments and treatment-delivery approaches coupled with a more appropriate personalized patient/regimen selection process [9].

It is difficult to fully appreciate the size of the unmet need as elderly and frail patients are less likely to be included in clinical trials and may receive fewer novel agents, partly as a consequence of comorbidities, polypharmacy and more rapid physiological decompensation associated with both disease- and treatment-related complications [5]. Furthermore, inferior outcomes may also be associated with a lack of a more personalised regimen selection. For example, similar outcomes have been reported in the $>75$ years old patient cohort in both the POLLUX and CASTOR trials [10]. Treatment strategies for patients deemed TNE have evolved and it is clear that improvements in survival have been less impressive in this group of patients [7, 11]. This is probably an under-estimation, in part, due to an underrepresentation of less fit patients in clinical trials due to strict entry criteria (see below) and a reflection of real-world practice where physicians may be less confident about delivering full dosing and delivery schedules in the more frail patient. It is likely that tolerance of full dose therapies, as mandated per protocol in these studies, is poor. The FIRST/MM020 trial recently demonstrated improved outcomes, including quality of life [12], with the IMiD, lenalidomide, continued to disease progression, over other treatment strategies [13]. Within this study patients' frailty index was assessed at baseline but doses were not prospectively adjusted according to frailty. Outcomes tended to improve for RD vs MPT in all frailty groups but with the greatest benefits were seen in fit patients [14, 15]. This suggests additional strategies are needed to improve outcomes for the unfit and frail groups. Furthermore, the lack of reliable international registry data limits a full-scale appreciation of the problem though national registries have contributed to our understanding of this unmet need. Current prognostic assessments rely on markers of either tumour burden (e.g. International Staging System; ISS) or genetic risk (FISH, mutational analysis, copy number variants) or a combination of both (R-ISS) [16, 17].

\section{Defining frailty}

Healthy ageing, and improving healthspan as well as prolonging lifespan are hugely important issues for both medicine and society in general. The difference between lifespan and healthspan, encompasses the influence of not only disease but of more rapid physiological deterioration, that predisposes to the consequence of concomitant illness and polypharmacy, further augmenting physiological decompensation-frailty. Frailty is a functional term that refers to a decline in physiological function, leading to dependency, vulnerability to stressors and high risk of health-related outcomes (metabolic disorders, infections, cancer) resulting in an increased morbidity and mortality [18]. Whilst the prevalence of frailty has been demonstrated to account for up to $60 \%$ of $>65$-year-old communitydwellers in western societies, all degrees of frailty have been reported in two-thirds of MM patients with severe frailty in at least $40 \%$ in some reported series [19, 20]. Two conceptual models of frailty exist - the Frailty Phenotype (often referred to frailty syndrome [21]) and Frailty Index [22], as illustrated in Fig. 1. The frailty phenotype,
Fig. 1 Defining frailty in suscetible populations. Two main accepted frailty assessment instruments $[14,15]$.

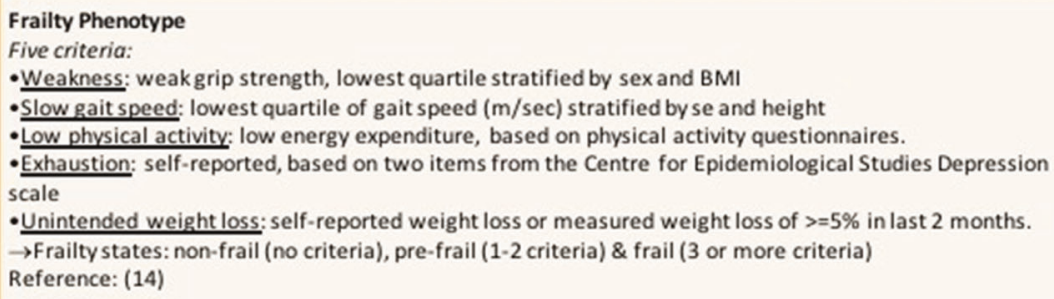

Reference:(15) 
recognising a link with sarcopenia, is defined as (1) unintentional weight loss, (2) weakness, (3) poor activity, (4) slowness of gait and (5) low physical activity level [21]. Sarcopenia, a progressive and generalised loss of skeletal muscle and function [23], is associated with the frailty phenotype [24]. The universally accepted definition of the functional sarcopenia was updated in 2019 by the European Working Group on Sarcopenia in older people (EWGSOP2). The definition included cut-offs to identify those who have sarcopenia. Biomarkers including imaging, to define the at risk population continues to evolve and may have a particular role in sarcopenic patients with cancer (see below).

\section{Clinical frailty scores}

The realization of the need for identifying populations with cancer who are at risk of therapy-related toxicity culminating in poorer outcomes consequential of limitations of systemic anti-cancer therapy (SACT) exposure prompted the publication of the American Society of Clinical Oncology guidance on use of geriatric assessments (GAs) and frailty scores in oncogeriatric practice [25]. In MM in patients deemed to be TNE there is considerable heterogeneity, not only in age but also the complex interplay of age, physical function, cognitive function and comorbidity. Furthermore, performance score (PS) and the ISS has been shown to be less able to discern these key sub-groups in the TNE population and predict their response to therapy and survivorship [7, 26]. A functional or GA offers the possible advantage of guiding therapeutic decisions with the potential to account for treatment compatibility, drug-induced side effects and mortality $[27,28]$. The use of a GA may complement a competent clinical judgement and indeed GA tools have been postulated to be valuable in a number of different cancers. However, generic GA tools have limitations and may not be applicable to patients with MM, with its well established disease-related morbidity (Fig. 2). The International Myeloma Working Group proposed a scoring system for MM patient frailty that predicts survival (IMWG FS), adverse events and treatment tolerability [29] using age, the Katz Activity of Daily Living (ADL), the Lawton Instrumental Activity of Daily Living (IADL) and the Charlson Comorbidity Index (CCI). The ADL ranks adequacy of performance in six functions: bathing, dressing, toileting, transferring, continence and feeding using a questionnaire-based tool. IADL assesses more complex activities (shopping, cooking and managing finances) necessary for functioning in community settings, the capacity to handle these complex functions are normally is lost before basic ADL. Both tools take 10-15 min to complete [30]. A pooled analysis of 869 patients with newly diagnosed MM who were being entered into several clinical intervention studies, and had a baseline frailty assessment using this scoring system demonstrated a correlation with survivorship. The IMWG FS may offer an additional clinical evaluation for the measurement of frailty, assisting both in the design and assessment of clinical interventional studies and perhaps, in day-to-day clinical practice. However, the IMWG FS was tested, but not validated, in the setting of clinical intervention studies and thus, patients are selected according to strict inclusion criteria, which may limit "realworld" interpretation of the data (see below). More recently, the IMWG FS was tested in an unselected real-world population outside of a clinical trial setting demonstrating it can be used to define a more biologically frail population [31].

As a consequence of this seminal work several scores have been developed and tested in various populations (summarized in Table 1) and a systematic review was conducted by Salazar et al., with some notable omissions [32]. Subsequent scores were developed that did not included the functionality of ADL and IADL, often using PS instead, especially for retrospectively developed scores. Facon et al. reported on a simplified frailty score using a
Fig. 2 The Frailty Spiral. The impact of aging, cellualr senescence and comorbidities on the evolution of frailty wiht the myeloma clinical features acting as "accelerants". The impact of multiple myeloma in accelerating age-related physiological decompensation (frailty).

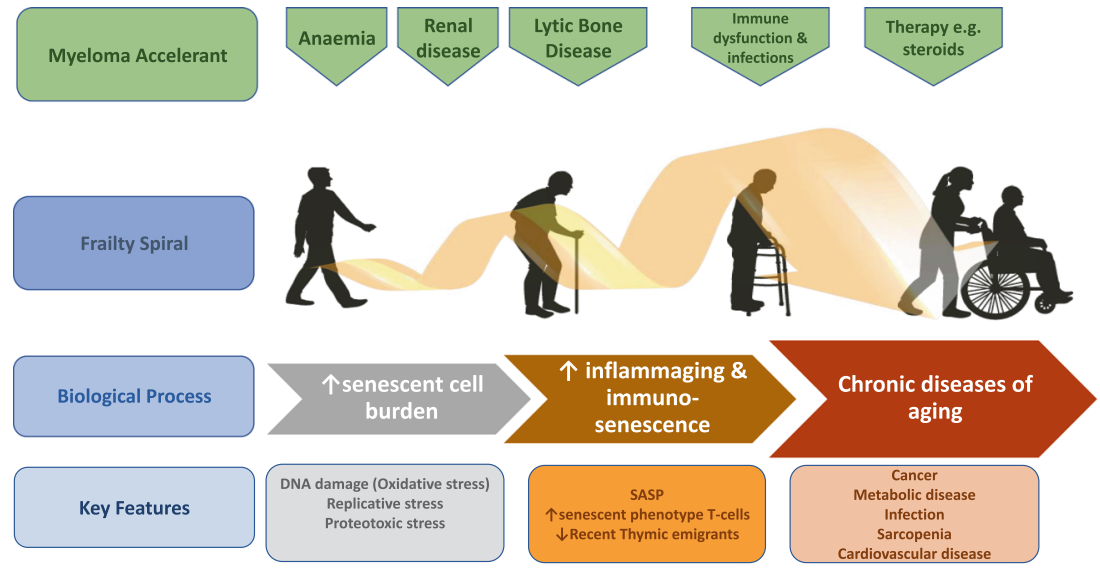


Table 1 Clinical frailty/risk scores in myeloma.

\begin{tabular}{|c|c|c|c|c|c|c|}
\hline Frailty score & $\begin{array}{l}\text { Biological } \\
\text { components }\end{array}$ & Functionality tests & $\begin{array}{l}\text { Comparison } \\
\text { with IMWG }\end{array}$ & Populations tested & $\begin{array}{l}\text { Prospective } \\
\text { evaluation? }\end{array}$ & Ref. \\
\hline IMWG & Age, CCI & ADL, IADL & - & CT & No & [29] \\
\hline R-MCI & $\begin{array}{l}\text { eGFR, PFTs, } \\
\text { Frailty, Age +/-CG }\end{array}$ & PS (Karnofsky) & Yes & $\mathrm{CT}, \mathrm{RW}$ & Yes & {$[20,33,35,42]$} \\
\hline UKMRA MRP & R-ISS, CRP, Age & PS (WHO) & No & $\mathrm{CT}, \mathrm{RW}$ & No & {$[37]$} \\
\hline Mayo Risk Score & NT-proBNP, Age & PS (WHO) & No & RW & No & {$[34]$} \\
\hline $\begin{array}{l}\text { Ancona } \\
\text { Vulnerability Score }\end{array}$ & CCI & PS (WHO) & No & RW & No & {$[36]$} \\
\hline
\end{tabular}

$C T$ clinical trials, $R W$ real world, $P S$ performance status, $A D L$ activities of daily living, $I A D L$ independent activities of daily living.

large clinical trial patient cohort $(n=1618)$, demonstrating that frail patients had inferior outcomes, especially overall survival. This simplified score was subsequently validated in an independent trial population [14, 15]. Engelhardt et al. prospectively assessed the impact of the IMWG FS on clinical outcome in a well-characterized non-trial cohort, comparing to alternative host-related scoring systems (R-MCI: revised Myeloma Comorbidity Index, CCI, KF index) and demonstrated the IMWG FS is a clinically useful tool in identifying patients with a host risk profile and is of prognostic value for functional decline and survivorship [33]. The Mayo Clinic developed a score (PS, Age and NTproBNP), assessed in just $<400$ consecutive patients [34]. The authors were able to define four clear sub-groups based on outcomes and in particular, defining OS differences between the groups ranging from 18 to 54 months $(p<$ $0.0001)$. One potential criticism of these single institutional studies developing models is that there is a tendency towards patient selection bias resulting from the tertiary nature of the authors clinical practice, albeit tertiary multicenter studies have just been prospectively shown to be feasible with different risk scores being compared [35]. Offidani et al. report a "vulnerability score" based on PS and CCI, though did test the impact of ISS, renal insufficiency and bone lesions as disease overlays [36]. Cook et al. generated a more laboratory-based objective risk score incorporating age, PS, CRP and ISS, which was able to discriminate not only therapy-related toxicity and regimen completion but survivorship and impact on quality of life [37]. Though this is more of a risk score than a traditional frailty score, it nonetheless defined patient populations who are vulnerable in the treatment setting. Similar to other scores reported here, it was tested and validated in clinical trial populations but has since been replicated in real-world setting [38].

A number of issues remain for clinical frailty assessment developments in MM before we can confidently rely on these. Firstly, the role of chronological age in scoring systems. Using age as a weighted factor is important but, to date, this has been subject to categorizing of what is in essence a continuous variable. This can leave individual patients switching from fit to unfit, or unfit to frail overnight as the age changes, which seems counter-intuitive when the frailty scores are after all measuring biological not chronological vulnerability. In addition, categorization can lead to an inflated type-1 statistical error, which increases the risk of a false-positive result in analysis [39]. As such, evolving our scores using age as a continuous variable seems appropriate. Secondly, in the IMWG FS, two measures that reflect activity are included (ADL and IADL), which in themselves are time-consuming and prone to subjectivity. Many of the other scoring systems have incorporated WHO PS (and one used Karnofsky PS) in replacement of the IMWG FS measures of activity, often as the models were developed using retrospective data, from which ADL and IADL cannot be implied as they were not collected contemporaneously [14, 33, 37]. Alternative approaches to find substitutes for the ADL/IADL have been described recently: using the FIRST trial data base, age, CCI and ECOG PS separated frail and fitter patients, which could define differences in outcomes (PFS, OS and treatment-related toxicities/endurance) [14]. Again, PS was used, but is more subjective, and prone to intra- and inter-observer bias and may not be able to detect disease burden overlay consequential to disease-related morbidity such as bone pain [40]. Given the limitations of these activity measures then we have an unmet need to more accurately define activity, and more specifically inactivity that is at the core of frailty. Given the development of wearable activity monitors, ironically most frequently used by younger fitter individuals for sport and leisure, then there is an opportunity to research the use of wearable devices to calibrate frailty susceptibility. One such study highlighted the use of stepping, walking and PAB parameters (sedentary and moderate-to-vigorous activity) in detecting pre-frailty [41]. Lastly, the use of the CCI has been core to many of the frailty scoring systems, though was not designed to be utilised in this setting in MM [20, 33, 35, 42]. Furthermore, given the average age of patients with MM and their documented multi-morbidity, this clinical setting represents the epitome of cluster 
medicine (Fig. 2). As such evolving the CCI to be more MM-specific may offer greater sensitivity when clinically assessing the impact of MM as a diagnosis as well as the risk of therapy intolerance. Engelhardt et al. devised a myeloma-specific comorbidity system that included 13 points of disease and organ dysfunction, in a similar design to the haematopoietic cell transplantation-specific comorbidity index [43], with the MCI preforming best in the dataset tested [20]. Further work can be useful in this regard [35, 42].

As the research evolves into frailty index mining, the key issues of validation before adoption include the need to compare to the accepted standard (IMWG FS), to test the prognostication prospectively, and to calibrate in a well-defined clinical trial populations before extending its predictability into real-world data is key. Some limited comparative studies and systematic reviews have been performed to date [32, 44, 45]. Salazar et al. presented a critical evaluation in a systematic review and metaanalysis. They identified seven studies, of which they only included three for the meta-analysis component [32]. However, they did not compare the scoring systems with IMWG FS rather they looked at the individual scoring system components and looked for efficacy of using frailty scoring in MM. It provides evidence that the use of such scoring systems is valid but not which one to adopt in clinical practice. Isaacs et al. [44] examined a comparison between IMWG FS, MCI and a cancer-based frailty deficit score (Carolina Frailty Score [46]), which as yet has not been tested in MM. Though this is to be applauded, the number of patients involved in the comparison is woefully inadequate to make a formal comparison valid. Formal validation of any score needs to included direct comparison with the IMWG FS to define that at least it proves the same level of discrepancy (null hypothesis-driven) before formally testing in prospective clinical trials and real-world data. Only then can its adoption in the clinic be warranted. Whether such scores can influence how a clinician delivers therapy (predictive biomarker) needs to be formally test in the clinical trial setting prospectively.

\section{Recommendations}

At this time, the IMWG FS remains the standard approach to defining frail and at risk populations in MM. Any and all developments in this setting need to be prospectively validated against the IMWG FS. Work needs to be done on refining the suitability, reproducibility and practical use of these systems as well as prospectively testing to define their prognostic biomarker potential. Only then can the predictive biomarker potential be tested in well-designed clinical trials. Alternative approaches, that have been compared and/or validated against the IMWG FS and include age, CCI, PS or other myeloma-appropriate risk factors could be clinically useful but such scoring systems need to be robust, reproducible and easy to use in the clinic.

\section{Biomarkers of frailty}

Gerosciene, the comprehensive multidisciplinary study of ageing and chronic disease in the older person, needs suitable and appropriate biomarkers to assess the ageing population and their risk of frailty syndrome (reviewed in [47]). This is especially important if the field is to expand into determining efficacy of interventions to improve healthspan and/or lifespan in a more preventative than curative medicine approach. In the context of MM, however, there are several issues. Firstly, we need to differentiate between biomarkers of ageing and those that reflect frailty. Much work is being done in this area (reviewed in [48]). Secondly, the use of high-throughput tools such as transcriptomics, proteomics, metabolomics whilst very important in advancing the biological understanding, in themselves are not suitable biomarkers for everyday use. However, understanding of the genetic and metabolic pathways may lead to an appropriate biomarker(s) for further everyday study for its value [49]. Nonetheless, such biomarkers need to be not only measurable but reliable, reproducible and feasible before validation in the clinical outcome setting. Thirdly, defining a biomarker as prognostic is not synonymous with prediction, which require further study in the setting of biomarker-driven clinical interventional studies [50]. Lastly, co-existing medical conditions of our patients as they are diagnosed can present as frailty and have a significant impact on treatment delivery and success. However, given that there is likely to be a disease-overlay (MM related morbidity) in the fitness of patient with age-related frailty, we need to have a dynamic assessment that highlights changes in frailty as treatment for MM proceeds. The clinical scales highlighted above are used as a static baseline consideration and have yet to prove sensitive enough to be a dynamic measure of susceptibility. As a consequence of these caveats, there is a need to develop and validate biomarkers of frailty in MM. The field continues to develop and is reviewed in [51]. It is worthy of note, that in developing biomarkers, either prognostic or predictive, we need to be cognoscente on whether biomarkers of frailty correspond to clinical measures of frailty, how do these biomarkers change with SACT, are frailty biomarkers correlative of patient-reported outcomes and can such biomarkers predict cancer-specific outcomes from SACT.

Several cellular pathways and biological functions lend themselves as potential areas for biomarker development. 
Table 2 Potential biomarkers of frailty.

\begin{tabular}{|c|c|c|c|}
\hline Category & Pathway & Biomarker(s) & Ref. \\
\hline \multirow[t]{2}{*}{ DNA associated } & Damage markers & $\gamma \mathrm{H} 2 \mathrm{AX}, \mathrm{ATM}, \mathrm{MDC} 1, \mathrm{TIF}$ & [71] \\
\hline & Telomere function & QuantPCR (length) Telomerase activity & {$[55,72]$} \\
\hline \multirow[t]{2}{*}{ Damage/repair } & Lysosomal activity & SA- $\beta$ Gal, $\alpha$-Fucos, Adiponectin & [73-75] \\
\hline & Circulating osteoprogenitor cells (COP) & $\mathrm{CD}_{4} / / \mathrm{CD} 45 / \mathrm{CD} 14 \% \mathrm{OCN}^{+}$Lamin A & [76] \\
\hline \multirow[t]{2}{*}{ Cell cycle related } & p16-pRB axis & $\mathrm{p} 16^{\mathrm{INK} 4 \mathrm{~A}}$ & [54] \\
\hline & p53-p21 axis & P53, RAS & [53] \\
\hline \multirow[t]{2}{*}{ Immunosenescence } & Component quantification & Senescent T-cells, Recent Thymic emigrants, $\mathrm{Th}_{17} / \mathrm{T}_{\mathrm{Reg}}$ cell ratios & {$[77,78]$} \\
\hline & SASP & IL-6, IL-8, IL-1, TNF $\alpha$, b-CHE, CRP, MiRNAs, eHsp72, selenium, Sirt1, PARP1 & {$[53,79]$} \\
\hline \multirow[t]{2}{*}{ Sarcopenia } & Imaging & DXA, CT, MRI & {$[64,80]$} \\
\hline & Blood biomarkers & Myostatin, ILGF-1 & {$[68,81,82]$} \\
\hline
\end{tabular}

Of these, three in particular hold promise for the field of host response biology in MM: cellular senescence, inflammaging/immunosenescence and sarcopenia. Current biomarkers of frailty are listed in Table 2 . Nearly 60 years ago, researchers identified the limited ability of human cells to divide, and in the intervening years, the cellular and molecular processes involved have been elucidated [52]. The current definition of senescence is characterized by three main features: arrested cell proliferation, resistance to apoptosis and the production of the senescence-associated secretory phenotype (SASP) [53]. Biomarkers of cellular senescence include markers of DNA damage $(\gamma \mathrm{H} 2 \mathrm{AX}$, ATM, MDC1), telomere length and telomere dysfunctioninduced foci (TIF), cell cycle arrest $\left(\mathrm{p} 16^{\mathrm{INK} 4 \mathrm{~A}}, \mathrm{p} 53 / \mathrm{p} 21\right.$ axis) and senescence-associated $\beta$-galactosidase (SA- $\beta \mathrm{Gal})$ $[54,55]$.

Inflammaging is a term generated 20 years ago when the role of the accumulation of age-dependent inflammatory mediators in cells and tissues resulting in low-grade, sterile and chronic inflammation is associated with the development of the frailty syndrome [56-58]. The concept being that pro-inflammatory mediators such as cytokines and chemokines play an essential causative role in the adversity associated with ageing.

Senescence of the immune system (immunosenescence) is one of the causes of inflammaging. Accordingly, a SASP has been described, which includes pro-inflammatory mediators such as IL-6, IL-8, IL-1, TNF $\alpha$, b-CHE, eHsp72, selenium and MicroRNAs (reviewed in [59]). In addition, alteration in immune cell subsets have been described including $\mathrm{Th}_{17} / \mathrm{T}_{\mathrm{Reg}}$ cell ratios, reduced recent thymic emigrants, $\mathrm{CD} 8^{+} \mathrm{CD} 28^{-} \mathrm{KLRG}-1^{+}$quantitation and dysfunctional $\mathrm{T}$-cell responses to TCR-mediated signals [60, 61]. The role of these immune component quantification studies and the relevance of measuring the SASP components in $\mathrm{MM}$ is yet to be determined but may represent peripheral blood accessible biomarkers for frailty (Table 2).

In accordance with the definition of sarcopenia (see above) as updated by the recent European Working Group on Sarcopenia in Older People (EWGSOP2), diagnosis requires the use of techniques to define a combination of appendicular skeletal muscle mass measurement $\left(\mathrm{kg} / \mathrm{m}^{2}\right)$, muscle strength usually defined by grip strength $(\mathrm{kg}$ or Newtons) and performance most commonly defined in clinic as gait speed $(\mathrm{m} / \mathrm{sec})$ or timed "Up and Go" test [23, 62]. Muscle mass can be measure by several techniques though none are ideal with major limitations. The most effective to date seems to be the dual-energy X-ray absorptiometry (DXA) but CT (especially Lumbar $3^{\text {rd }}$ vertebra by CT imaging in cancer patients including MM [63]) and more recently MRI scanning have been advocated, the latter being able to pose a calibration of muscle quality as well as mass $[62,64,65]$. One promising approach is the $d 3$ creatinine $\mathrm{A}$ isotope dilution test, which has generated more correlative results with sarcopenia than DXA [66]. In MM context, early results from the HOVON 123 study have defined the predictive value of the IMWG FS and loss of muscle mass in frail with the reported outcomes included treatment tolerability [67]. Interestingly, the authors report that low muscle mass rather than muscle function were associated with clinical outcomes.

Blood-based biomarkers of sarcopenia are still in the developmental stages and have proven thus far to be more complicated to define than perhaps first thought, especially the relationship between adipocyte metabolism, turn-over and senescence with sarcopenia. Potential biomarkers include myostatin, Insulin-like Growth Factor 1, as well as markers of inflammation [68] but to date imaging currently offers the best measure of sarcopenia and outcomes in cancer patients.

\section{Recommendations}

Biomarker discovery to better define frailty syndrome is evolving in internal medicine but is very much in its infancy on Oncogeriatrics. In relation to MM, such biomarkers have yet to be systematically studied. As such we recommend that further study of their importance is needed in clinically defined trial propulsions before any such biomarkers can be 
recommended for prognostic determination let alone function as predictive biomarkers.

\section{Validation of clinical scores and biomarkers}

To date, the clinical frailty scores described above, have been largely developed using clinical trial datasets for both hypothesis testing and model validation or retrospective single-centre real-world populations. However, clinical trial datasets represent the perfect data in the imperfect population compared with real-world data, which represents the imperfect data in the perfect patient in respect to how we deliver everyday care in the clinic. For example, Shah et al. found that using common randomized controlled trial (RCT) eligibility criteria, only $60 \%$ of patients in the realworld were eligible for participation in RCT, in part related to renal function and lower hematopoietic reserve [69]. As a consequence patients ineligible for RCTs demonstrated more advanced disease (Connect MM Registry ISS III $22.1 \%$ in RCT eligible versus $40.1 \%$ in RCT ineligible; $p<$ 0.001). NCRI Myeloma XI, the largest upfront trial in newly diagnosed MM, had a median age of 74 in the TNE cohort though only $13.2 \%$ were older than 80 years compared with the population rate of MM in the UK where $54.8 \%$ are older than 80 years of age [7]. As such it is clear that any clinical scoring system or biomarker, whether prognostic or predictive needs to be evaluated in a realworld population if the true impact on everyday clinical practice is to be measured $[20,33,35,42,70]$.

\section{Future directions}

It is clear from the current evidence and the stated unmet need that the potential of GA tools in assessing TNE MM patients and influence healthcare delivery is important.
However that one represents the best and most responsive tool remains to be defined. Given the unmet need and the potential for the clinical utility of a GA tool to predict survivorship, the next step is to test whether such a tool can be used to direct treatment delivery. In this context, the UK Myeloma Research Alliance (UKMRA) has developed the Myeloma XIV: FITNESS study (NCT03720041). In this study, patients will be randomized to a treatment-adaptive arm where therapy will be dose-reduced in accordance with the IMWG frailty score compared with a conventional treatment-reactive arm where therapy will be modified in relation to toxicity and tolerance (https://clinicaltrials.gov/ ct $2 /$ show/NCT03720041 cond $=$ myeloma + XIV \&draw $=$

$2 \&$ rank $=1$ ). The aim of the study will be the development and assessment of a host biological scoring tool to predict treatment tolerance, prevention of treatment discontinuation and reduction of early death as well as defining the impact on PFS and survivorship. Funded by Cancer Research UK, the trial has opened for recruitment in March 2020. This is one approach to the use of GA tools by defining who will not benefit from standard of care. Another direction of clinical research is to highlight this population using GA tools, and provide alternative treatment regimens and delivery. Some novel agents are more suitable to such frail populations. Furthermore, we need to study how dynamic GA tools are, how frailty may change over time (age-related vs disease-related frailty) and the impact of novel interventions to may be adjuvant to the delivery of primary antimyeloma therapy. There are currently seven other frailtyassociated trials in MM listed on clintrials.gov either recruiting or in set up (https://clinicaltrials.gov/ct2/results? cond $=$ Myeloma\&term $=$ frailty $\&$ cntry $=\&$ state $=\&$ city $=$ \&dist=) and a list of key trials of interest are listed in Table 3.

The inclusion of clinical frailty scores in everyday practice is best facilitated through tumour boards, where case-by-case discussion about treatment pathways and

Table 3 Selected prospective clinical trials in MM, adapted from [42].

\begin{tabular}{|c|c|c|c|c|c|}
\hline Trial Collaborative/Institution & Trial & Phase & Trial designation & Patient cohort & Study details \\
\hline University of Freiburg & VBDD [83] & $\mathrm{I} / \mathrm{II}$ & NCT01394354 & 33 & $\begin{array}{l}\text { QoL \& frailty improvements } \\
\text { in disease responsiveness }\end{array}$ \\
\hline City of Hope/ University of Rochester & Touch-screen based GA & IV & NCT03068637 & 165 & $\begin{array}{l}\text { Utility of technology to } \\
\text { improve clinic applicability of } \\
\text { GA usage }\end{array}$ \\
\hline GIMEMA & Rd vs Rd-R in unfit MM [84] & II & NCT02215980 & 210 & $\begin{array}{l}\text { Rd-R improved outcomes for } \\
\text { unfit patients }\end{array}$ \\
\hline HOVON & $\begin{array}{l}\text { HOVON 143: Efficacy \& } \\
\text { tolerability of IDd in unfit \& } \\
\text { frail patients }\end{array}$ & II & NCR6297 & 130 & $\begin{array}{l}\text { Dose-adjustments feasible } \\
\text { though effect on early } \\
\text { mortality still to be defined }\end{array}$ \\
\hline UKMRA & $\begin{array}{l}\text { UKMRA Myeloma XIV } \\
\text { (FiTNEss) trial: Use of frailty } \\
\text { scores to direct clinical } \\
\text { treatment adjustments (IRD) } \\
\text { in TNE NDMM. }\end{array}$ & III & NCT03720041 & 740 & $\begin{array}{l}\text { Frailty-adapted versus } \\
\text { toxicity-responsive dose } \\
\text { adjustments and impact on } \\
\text { outcomes including early } \\
\text { mortality }\end{array}$ \\
\hline
\end{tabular}

QoL quality of life, GA geriatric assessment, $R d$ lenalidomide and dexamethasone, IDd ixazomib, daratumumab \& dexamethasone, IRD ixazomib, lenalidomide \& dexamethasone, $T N E$ - transplant ineligible, $N D M M$ new diagnosed MM. 
management are held. However, at present, clinical frailty scores have been proven as prognostic biomarkers, but as yet have not been proven as predictive biomarkers. It is only when defined as predictive biomarkers, can clinical confidence be ensured in patient decision making and the above outline clinical trials aim to define this.

\section{Conclusions}

As the treatment landscape continues to evolve towards the application of precision medicine in MM, there is a clear need to take stock of the host response biology when designing therapeutic strategies to maximize efficacy whilst minimizing toxicity and ensuring the best possible treatment delivery. An integral aspect of this approach is to define the physiological age and capacity of patients with MM to deal with the burden of their disease and it's treatment. Such assessments may include not only functional and clinical assessments but also laboratory-based biomarkers of frailty, aging and senescent cellular burden. A need to develop, test and validate clinical screening scores before their adoption into clinical practice is mandated. Ongoing research into potential biomarkers of susceptibility and frailty may yield more time-sensitivity markers of frailty. Hopefully we are approaching a time where such measurements, once validated prospectively, will be used to direct safe, effective and personalized treatment.

Author contributions GC, AL, SZ, TF and ME designed the manuscript content and GC wrote the first draft. GC, AL, SZ, TF and ME edited and produced the final draft for submission.

\section{Compliance with ethical standards}

Conflict of interest GC-Honoraria: Amgen, Bristol-Myers Squibb, Celgene, Janssen, Sanofi, Karyopharm and GSK; Research funding: Celgene, Janssen, Takeda. AL-Honoraria: Amgen, Bristol-Myers Squibb, Celgene, Janssen and GSK; Advisory Board: Bristol-Myers Squibb, Celgene, Janssen, Takeda. SZ-Advisory board: Celgene, Janssen, Takeda, Sanofi, Oncopeptides; Research support: Celgene, Janssen, Takeda. TF and ME—no relevant conflicts.

Publisher's note Springer Nature remains neutral with regard to jurisdictional claims in published maps and institutional affiliations.

\section{References}

1. Pilleron S, Sarfati D, Janssen-Heijnen M, Vignat J, Ferlay J, Bray F, et al. Global cancer incidence in older adults, 2012 and 2035: a population-based study. Int J Cancer. 2019;144:49-58.

2. Palumbo A, Bringhen S, Ludwig H, Dimopoulos MA, Bladé J, Mateos MV, et al. Personalized therapy in multiple myeloma according to patient age and vulnerability: a report of the European Myeloma Network (EMN). Blood. 2011; 118:4519-29.
3. Cowan AJ, Allen C, Barac A, Basaleem H, Bensenor I, Curado MP, et al. Global burden of multiple myeloma: a systematic analysis for the global burden of disease study 2016. JAMA Oncol. 2018;4:1221-7.

4. Mellqvist U-H. New prognostic tools for myeloma. Blood. 2015;125:2014-5.

5. Bringhen S, Mateos MV, Zweegman S, Larocca A, Falcone AP, Oriol A, et al. Age and organ damage correlate with poor survival in myeloma patients: meta-analysis of 1435 individual patient data from 4 randomized trials. Haematologica. 2013;98:980-7.

6. Kint N, Delforge M. Concise review-treatment of multiple myeloma in the very elderly: how do novel agents fit in? J Geriatr Oncol. 2016;7:383-9.

7. Pawlyn C, Cairns D, Kaiser M, Striha A, Jones J, Shah V, et al. The relative importance of factors predicting outcome for myeloma patients at different ages: results from 3894 patients in the Myeloma XI trial. Leukemia. 2020;34:604-12.

8. Walter LC, Covinsky KE. Cancer screening in elderly patients: a framework for individualized decision making. JAMA. 2001;285:2750-6.

9. Zweegman S, Larocca A. Frailty in multiple myeloma: the need for harmony to prevent doing harm. Lancet Haematol. 2019;6: e117-e118.

10. Mateos M-V, Spencer A, Nooka AK, Pour L, Weisel K, Cavo M, et al. Daratumumab-based regimens are highly effective and well tolerated in relapsed or refractory multiple myeloma regardless of patient age: subgroup analysis of the phase 3 CASTOR and POLLUX studies. Haematologica. 2020;105:468-77.

11. Pulte D, Jansen L, Castro FA, Emrich K, Katalinic A, Holleczek $\mathrm{B}$, et al. Trends in survival of multiple myeloma patients in Germany and the United States in the first decade of the 21st century. Br J Haematol. 2015;171:189-96.

12. Delforge M, Minuk L, Eisenmann J-C, Arnulf B, Canepa L, Fragasso A, et al. Health-related quality-of-life in patients with newly diagnosed multiple myeloma in the FIRST trial: lenalidomide plus low-dose dexamethasone versus melphalan, prednisone, thalidomide. Haematologica. 2015;100:826-33.

13. Benboubker L, Dimopoulos MA, Dispenzieri A, Catalano J, Belch $\mathrm{AR}$, Cavo $\mathrm{M}$, et al. Lenalidomide and dexamethasone in transplant-ineligible patients with myeloma. N Engl J Med. 2014; 371:906-17.

14. Facon T, Dimopoulos MA, Meuleman N, Belch A, Mohty M, Chen W-M, et al. A simplified frailty scale predicts outcomes in transplant-ineligible patients with newly diagnosed multiple myeloma treated in the FIRST (MM-020) trial. Leukemia. 2020;34:224-33.

15. Stege CAM, van der Holt B, Dinmohamed AG, Sonneveld P, Levin M-D, van de Donk NWCJ, et al. Validation of the FIRST simplified frailty scale using the ECOG performance status instead of patient-reported activities. Leukemia. 2020. https://doi.org/10. 1038/s41375-020-0713-4.

16. Palumbo A, Avet-Loiseau H, Oliva S, Lokhorst HM, Goldschmidt $\mathrm{H}$, Rosinol L, et al. Revised international staging system for multiple myeloma: a report from international myeloma working group. J Clin Oncol. 2015;33:2863-9.

17. Facon T, Anderson K. Treatment approach for the older, unfit patient with myeloma from diagnosis to relapse: perspectives of a European hematologist. Hematology Am Soc Hematol Educ Program. 2018;2018:83-87.

18. Fried LP, Hadley EC, Walston JD, Newman AB, Newman A, Guralnik JM, et al. From bedside to bench: research agenda for frailty. Sci Aging Knowledge Environ. 2005;2005:pe24.

19. Collard RM, Boter H, Schoevers RA, Oude Voshaar RC. Prevalence of frailty in community-dwelling older persons: a systematic review. J Am Geriatr Soc. 2012;60:1487-92. 
20. Engelhardt M, Domm A-S, Dold SM, Ihorst G, Reinhardt H, Zober A, et al. A concise revised Myeloma Comorbidity Index as a valid prognostic instrument in a large cohort of 801 multiple myeloma patients. Haematologica. 2017;102:910-21.

21. Fried LP, Tangen CM, Walston J, Newman AB, Hirsch C, Gottdiener J, et al. Frailty in older adults: evidence for a phenotype. J Gerontol A Biol Sci Med Sci. 2001;56:M146-156.

22. Rockwood K, Mitnitski A. Frailty in relation to the accumulation of deficits. J Gerontol A Biol Sci Med Sci. 2007;62:722-7.

23. Cruz-Jentoft AJ, Bahat G, Bauer J, Boirie Y, Bruyère O, Cederholm T, et al. Sarcopenia: revised European consensus on definition and diagnosis. Age Ageing. 2019;48:601.

24. Cesari M. The frailty phenotype and sarcopenia: similar but not the same. Aging Med (Milton). 2019;2:97-98.

25. Mohile SG, Dale W, Somerfield MR, Schonberg MA, Boyd CM, Burhenn PS, et al. Practical assessment and management of vulnerabilities in older patients receiving chemotherapy: ASCO guideline for geriatric oncology. J Clin Oncol. 2018;36:2326-47.

26. Kuroda J, Shimura Y, Ohta K, Tanaka H, Shibayama H, Kosugi S, et al. Limited value of the international staging system for predicting long-term outcome of transplant-ineligible, newly diagnosed, symptomatic multiple myeloma in the era of novel agents. Int J Hematol. 2014;99:441-9.

27. Wildes TM, Campagnaro E. Management of multiple myeloma in older adults: gaining ground with geriatric assessment. J Geriatr Oncol. 2017;8:1-7.

28. Wildes TM, Tuchman SA, Klepin HD, Mikhael J, Trinkaus K, Stockerl-Goldstein K, et al. Geriatric assessment in older adults with multiple myeloma. J Am Geriatr Soc. 2019;67:987-91.

29. Palumbo A, Bringhen S, Mateos M-V, Larocca A, Facon T, Kumar SK, et al. Geriatric assessment predicts survival and toxicities in elderly myeloma patients: an International Myeloma Working Group report. Blood. 2015;125:2068-74.

30. Kojima G. Quick and simple FRAIL scale predicts incident activities of daily living (ADL) and instrumental ADL (IADL) disabilities: a systematic review and meta-analysis. J Am Med Dir Assoc. 2018;19:1063-8.

31. Mina R, Bringhen S, Wildes TM, Zweegman S, Rosko AE. Approach to the older adult with multiple myeloma. Am Soc Clin Oncol Educ Book. 2019;39:500-18.

32. Salazar AS, Recinos LM, Mian HS, Stoll C, Simon LE, Sekhon S, et al. Geriatric assessment and frailty scores predict mortality in myeloma: systematic review and meta-analysis. Clin Lymphoma Myeloma Leuk. 2019;19:488-496.e6.

33. Engelhardt M, Dold SM, Ihorst G, Zober A, Möller M, Reinhardt $\mathrm{H}$, et al. Geriatric assessment in multiple myeloma patients: validation of the International Myeloma Working Group (IMWG) score and comparison with other common comorbidity scores. Haematologica. 2016;101:1110-9.

34. Milani P, Vincent Rajkumar S, Merlini G, Kumar S, Gertz MA, Palladini G, et al. N-terminal fragment of the type-B natriuretic peptide (NT-proBNP) contributes to a simple new frailty score in patients with newly diagnosed multiple myeloma. Am J Hematol. 2016;91:1129-34.

35. Dold SM, Möller M-D, Ihorst G, Langer C, Pönisch W, Mügge L$\mathrm{O}$ et al. Validation of the revised myeloma comorbidity index and other comorbidity scores in a multicenter German study group multiple myeloma trial. Haematologica. 2020. https://doi.org/10. 3324/haematol.2020.254235.

36. Offidani M, Corvatta L, Polloni C, Centurioni R, Visani G, Brunori $\mathrm{M}$, et al. Assessment of vulnerability measures and their effect on survival in a real-life population of multiple myeloma patients registered at Marche Region Multiple Myeloma Registry. Clin Lymphoma Myeloma Leuk. 2012;12:423-32.

37. Cook G, Royle K-L, Pawlyn C, Hockaday A, Shah V, Kaiser MF, et al. A clinical prediction model for outcome and therapy delivery in transplant-ineligible patients with myeloma (UK Myeloma Research Alliance Risk Profile): a development and validation study. Lancet Haematol. 2019;6:e154-e166.

38. Redder L, Klausen TW, Vangsted AJ, Gregersen H, Andersen NF, Pedersen RS, et al. Validation of a new clinical prediction model for outcome in newly diagnosed multiple myeloma patients not eligible for autologous stem-cell transplantation; a populationbased study from the danish national multiple myeloma registry. Blood. 2019;134:1849-1849.

39. Barnwell-Ménard J-L, Li Q, Cohen AA. Effects of categorization method, regression type, and variable distribution on the inflation of Type-I error rate when categorizing a confounding variable. Stat Med. 2015;34:936-49.

40. Giri S, Williams G, Rosko A, Grant SJ, Mian HS, Tuchman S, et al. Simplified frailty assessment tools: are we really capturing frailty or something else? Leukemia. 2020. https://doi.org/10. 1038/s41375-020-0712-5.

41. Razjouyan J, Naik AD, Horstman MJ, Kunik ME, Amirmazaheri $\mathrm{M}$, Zhou $\mathrm{H}$, et al. Wearable sensors and the assessment of frailty among vulnerable older adults: an observational cohort study. Sensors (Basel). 2018;18. https://doi.org/10.3390/s18051336.

42. Engelhardt M, Ihorst G, Duque-Afonso J, Wedding U, SpätSchwalbe E, Goede V, et al. Structured assessment of frailty in multiple myeloma as a paradigm of individualized treatment algorithms in cancer patients at advanced age. Haematologica. 2020;105:1183-8.

43. Sorror ML, Maris MB, Storb R, Baron F, Sandmaier BM, Maloney DG, et al. Hematopoietic cell transplantation (HCT)-specific comorbidity index: a new tool for risk assessment before allogeneic HCT. Blood. 2005;106:2912-9.

44. Isaacs A, Fiala M, Tuchman S, Wildes TM. A comparison of three different approaches to defining frailty in older patients with multiple myeloma. J Geriatr Oncol. 2020;11:311-5.

45. Mian H, Brouwers M, Kouroukis CT, Wildes TM. Comparison of frailty scores in newly diagnosed patients with multiple myeloma: a review. J Frailty Aging. 2019;8:215-21.

46. Guerard EJ, Deal AM, Chang Y, Williams GR, Nyrop KA, Pergolotti M, et al. Frailty index developed from a cancer-specific geriatric assessment and the association with mortality among older adults with cancer. J Natl Compr Canc Netw. 2017;15: 894-902.

47. Campisi J, Kapahi P, Lithgow GJ, Melov S, Newman JC, Verdin E. From discoveries in ageing research to therapeutics for healthy ageing. Nature. 2019;571:183-92.

48. Saedi AA, Feehan J, Phu S, Duque G. Current and emerging biomarkers of frailty in the elderly. Clin Interv Aging. 2019;14: 389-98.

49. Muscedere J, Kim PM, Afilalo J, Balion C, Baracos VE, Bowdish $\mathrm{D}$, et al. Proceedings of the Canadian frailty network workshop: identifying biomarkers of frailty to support frailty risk assessment, diagnosis and prognosis. Toronto, January 15, 2018. J Frailty Aging. 2019;8:106-16.

50. Oldenhuis CNaM, Oosting SF, Gietema JA, de Vries EGE. Prognostic versus predictive value of biomarkers in oncology. Eur J Cancer. 2008;44:946-53.

51. Picca A, Calvani R. Biomarkers of frailty: moving the field forward. Exp Gerontol. 2020;133:110868.

52. Hayflick L, Moorhead PS. The serial cultivation of human diploid cell strains. Exp Cell Res. 1961;25:585-621.

53. Coppé J-P, Patil CK, Rodier F, Sun Y, Muñoz DP, Goldstein J, et al. Senescence-associated secretory phenotypes reveal cellnonautonomous functions of oncogenic RAS and the p53 tumor suppressor. PLoS Biol. 2008;6:2853-68.

54. Matjusaitis M, Chin G, Sarnoski EA, Stolzing A. Biomarkers to identify and isolate senescent cells. Ageing Res Rev. 2016;29: $1-12$. 
55. Zhou J, Wang J, Shen Y, Yang Y, Huang P, Chen S, et al. The association between telomere length and frailty: a systematic review and meta-analysis. Exp Gerontol. 2018;106:16-20.

56. Franceschi C, Zaikin A, Gordleeva S, Ivanchenko M, Bonifazi F, Storci G, et al. Inflammaging 2018: an update and a model. Semin Immunol. 2018;40:1-5.

57. Fulop T, Larbi A, Dupuis G, Le Page A, Frost EH, Cohen AA, et al. Immunosenescence and inflamm-aging as two sides of the same coin: friends or foes? Front Immunol. 2017;8:1960.

58. Franceschi C, Bonafè M, Valensin S, Olivieri F, De Luca M, Ottaviani E, et al. Inflamm-aging. An evolutionary perspective on immunosenescence. Ann NY Acad Sci. 2000;908:244-54.

59. Vatic M, von Haehling S, Ebner N. Inflammatory biomarkers of frailty. Exp Gerontol. 2020;133:110858.

60. Fulop T, McElhaney J, Pawelec G, Cohen AA, Morais JA, Dupuis $\mathrm{G}$, et al. Frailty, inflammation and immunosenescence. Interdiscip Top Gerontol Geriatr. 2015;41:26-40.

61. McElhaney JE, Zhou X, Talbot HK, Soethout E, Bleackley RC, Granville DJ, et al. The unmet need in the elderly: how immunosenescence, CMV infection, co-morbidities and frailty are a challenge for the development of more effective influenza vaccines. Vaccine. 2012;30:2060-7.

62. Cruz-Jentoft AJ, Sayer AA. Sarcopenia. Lancet. 2019;393: 2636-46.

63. Zakaria HM, Llaniguez JT, Telemi E, Chuang M, Abouelleil M, Wilkinson B, et al. Sarcopenia predicts overall survival in patients with lung, breast, prostate, or myeloma spine metastases undergoing stereotactic body radiation therapy (sbrt), independent of histology. Neurosurgery. 2020;86:705-16.

64. Codari M, Zanardo M, di Sabato ME, Nocerino E, Messina C, Sconfienza LM, et al. MRI-derived biomarkers related to sarcopenia: a systematic review. J Magn Reson Imaging. 2020;51: $1117-27$.

65. Khan AI, Reiter DA, Sekhar A, Sharma P, Safdar NM, Patil DH, et al. MRI quantitation of abdominal skeletal muscle correlates with CT-based analysis: implications for sarcopenia measurement. Appl Physiol Nutr Metab. 2019;44:814-9.

66. Cawthon PM, Orwoll ES, Peters KE, Ensrud KE, Cauley JA, Kado DM, et al. Strong relation between muscle mass determined by D3-creatine dilution, physical performance, and incidence of falls and mobility limitations in a prospective cohort of older men. J Gerontol A Biol Sci Med Sci. 2019;74:844-52.

67. Stege CAM, Nasserinejad K, Levin M-D, Klein SK, Waal E, de, Eeltink $C$, et al. Geriatric impairments and low muscle mass are associated with treatment discontinuation and overall survival in newly diagnosed non-transplant eligible multiple myeloma patients (nte-NDMM) treated with dose-adjusted melphalanprednisone-bortezomib (MPV)—results of the Dutch HOVON 123 study. Blood. 2018;132:1889-1889.

68. Chew J, Tay L, Lim JP, Leung BP, Yeo A, Yew S, et al. Serum myostatin and IGF-1 as gender-specific biomarkers of frailty and low muscle mass in community-dwelling older adults. J Nutr Health Aging. 2019;23:979-86.

69. Shah JJ, Abonour R, Gasparetto C, Hardin JW, Toomey K, Narang M, et al. Analysis of common eligibility criteria of randomized controlled trials in newly diagnosed multiple myeloma patients and extrapolating outcomes. Clin Lymphoma Myeloma Leuk. 2017;17:575-583.e2.

70. Gregersen H, Vangsted AJ, Abildgaard N, Andersen NF, Pedersen RS, Frølund UC, et al. The impact of comorbidity on mortality in multiple myeloma: a Danish nationwide population-based study. Cancer Med. 2017;6:1807-16.

71. Valdiglesias V, Sánchez-Flores M, Marcos-Pérez D, LorenzoLópez L, Maseda A, Millán-Calenti JC, et al. Exploring genetic outcomes as frailty biomarkers. J Gerontol A Biol Sci Med Sci. 2019;74:168-75.

72. Woo J, Tang NLS, Suen E, Leung JCS, Leung PC. Telomeres and frailty. Mech Ageing Dev. 2008;129:642-8.

73. Nagasawa M, Takami Y, Akasaka H, Kabayama M, Maeda S, Yokoyama S, et al. High plasma adiponectin levels are associated with frailty in a general old-old population: The Septuagenarians, Octogenarians, Nonagenarians Investigation with Centenarians study. Geriatr Gerontol Int. 2018;18:839-46.

74. Tsai J-S, Wu C-H, Chen S-C, Huang K-C, Chen C-Y, Chang C-I, et al. Plasma adiponectin levels correlate positively with an increasing number of components of frailty in male elders. PLoS ONE. 2013;8:e56250.

75. Kurz DJ, Decary S, Hong Y, Erusalimsky JD. Senescenceassociated (beta)-galactosidase reflects an increase in lysosomal mass during replicative ageing of human endothelial cells. J Cell Sci. 2000;113(Pt 20):3613-22.

76. Gunawardene P, Al Saedi A, Singh L, Bermeo S, Vogrin S, Phu S, et al. Age, gender, and percentage of circulating osteoprogenitor (COP) cells: the COP study. Exp Gerontol. 2017;96:68-72.

77. Yin M-J, Xiong Y-Z, Xu X-J, Huang L-F, Zhang Y, Wang X-J, et al. Tfh cell subset biomarkers and inflammatory markers are associated with frailty status and frailty subtypes in the community-dwelling older population: a cross-sectional study. Aging (Albany NY). 2020;12:2952-73.

78. Duggal NA, Pollock RD, Lazarus NR, Harridge S, Lord JM. Major features of immunesenescence, including reduced thymic output, are ameliorated by high levels of physical activity in adulthood. Aging Cell. 2018;17. https://doi.org/10.1111/acel. 12750.

79. Hekmatimoghaddam S, Dehghani Firoozabadi A, Zare-Khormizi MR, Pourrajab F. Sirt1 and Parp1 as epigenome safeguards and microRNAs as SASP-associated signals, in cellular senescence and aging. Ageing Res Rev. 2017;40:120-41.

80. Portal D, Hofstetter L, Eshed I, Dan-Lantsman C, Sella T, Urban $\mathrm{D}$, et al. L3 skeletal muscle index (L3SMI) is a surrogate marker of sarcopenia and frailty in non-small cell lung cancer patients. Cancer Manag Res. 2019;11:2579-88.

81. Kwak JY, Hwang H, Kim S-K, Choi JY, Lee S-M, Bang H, et al. Prediction of sarcopenia using a combination of multiple serum biomarkers. Sci Rep. 2018;8:8574.

82. Boreskie KF, Oldfield CJ, Hay JL, Moffatt TL, Hiebert BM, Arora RC, et al. Myokines as biomarkers of frailty and cardiovascular disease risk in females. Exp Gerontol. 2020; 133:110859.

83. Waldschmidt JM, Keller A, Ihorst G, Grishina O, Müller S, Wider $\mathrm{D}$, et al. Safety and efficacy of vorinostat, bortezomib, doxorubicin and dexamethasone in a phase I/II study for relapsed or refractory multiple myeloma (VERUMM study: vorinostat in elderly, relapsed and unfit multiple myeloma). Haematologica. 2018;103:e473-e479.

84. Larocca A, Salvini M, De Paoli L, Cascavilla N, Benevolo G, Galli M, et al. Efficacy and feasibility of dose/schedule-adjusted $\mathrm{Rd}-\mathrm{R}$ vs. continuous Rd in elderly and intermediate-fit newly diagnosed multiple myeloma (NDMM) patients: RV-MM-PI0752 phase III randomized study. Blood. 2018;132:305-305. 